Projets

de paysage

\section{Projets de paysage}

Revue scientifique sur la conception et l'aménagement de l'espace

$8 \mid 2012$

Les concepteurs de jardins et de parcs japonais

\title{
La gestion des espaces ouverts de Lille Métropole
}

Produire un " paysage vivant », mettre à contribution l'agriculture

Managing the open spaces of Lille Métropole - Producing a "living landscape", calling upon agriculture

\section{Nicolas Rouget}

\section{OpenEdition}

\section{Journals}

Édition électronique

URL : http://journals.openedition.org/paysage/15344

DOI : $10.4000 /$ paysage. 15344

ISSN : 1969-6124

\section{Éditeur :}

École nationale supérieure du paysage de Versailles-Marseille, Institut national des sciences appliquées Centre Val de Loire - École de la nature et du paysage, École nationale supérieure d'architecture et de paysage de Bordeaux, École nationale supérieure d'architecture et de paysage de Lille, Agrocampus Angers

Référence électronique

Nicolas Rouget, «La gestion des espaces ouverts de Lille Métropole », Projets de paysage [En ligne], 8 | 2012, mis en ligne le 13 juillet 2012, consulté le 11 mars 2021. URL : http://journals.openedition.org/ paysage/15344; DOI : https://doi.org/10.4000/paysage.15344

Ce document a été généré automatiquement le 11 mars 2021

Projets de paysage 


\title{
La gestion des espaces ouverts de Lille Métropole
}

\author{
Produire un « paysage vivant », mettre à contribution l'agriculture \\ Managing the open spaces of Lille Métropole - Producing a "living landscape", \\ calling upon agriculture
}

Nicolas Rouget

1 Les discours et les documents de planification lillois ont pour leitmotiv le concept de «ville renouvelée ». Ce concept implique une redensification du tissu urbain mais aussi la volonté d'inscrire la «nature » au sein du territoire de la cité.

2 Cette «nature » est assimilée à une "nouvelle campagne », espace rural devenu cadre paysager de la ville (Poulot, Rouyres, 2007).

3 La délimitation de parcs périurbains (image 1), définis en tant que territoires d'intervention du syndicat mixte ENLM $^{1}$, doit contribuer à l'émergence de cette «nouvelle campagne». L'objectif du syndicat mixte est celui d'un "paysage vivant, ouvert à la promenade et économiquement faisable, donc en collaboration avec les agriculteurs ${ }^{2} »$ (Pierre Dhénin, directeur ENLM, réunion de présentation du partenariat ville-agriculture aux exploitants du Val de Marque, 2005).

4 L'étude du processus par lequel a été rendu possible, à Lille, le rapprochement des sphères agricoles et des sphères politiques citadines est un moyen pour décrire et comprendre comment et à quelles conditions se met en place une convergence des représentations de l'espace et des intérêts entre acteurs du territoire. Pourquoi et comment s'est opéré ce rapprochement entre décideurs urbains et acteurs agricoles? Quels sont les fondements et quelle est la nature du partenariat proposé par l'ENLM aux exploitants agricoles?

5 L'exemple lillois pose également la question de l'efficience des expériences menées en matière de gestion participative (Biasi, Pujol, 2005 ; Aubertin, Pinton, Rodary, 2008). Quels sont les résultats des politiques de mise à contribution de l'agriculture conduites au sein des parcs de la Deûle et du Val de Marque, parcs inscrits comme prioritaires au schéma directeur de l'arrondissement de Lille? 
Figure 1. Les territoires d'intervention du syndicat mixte Espace naturel Lille Métropole

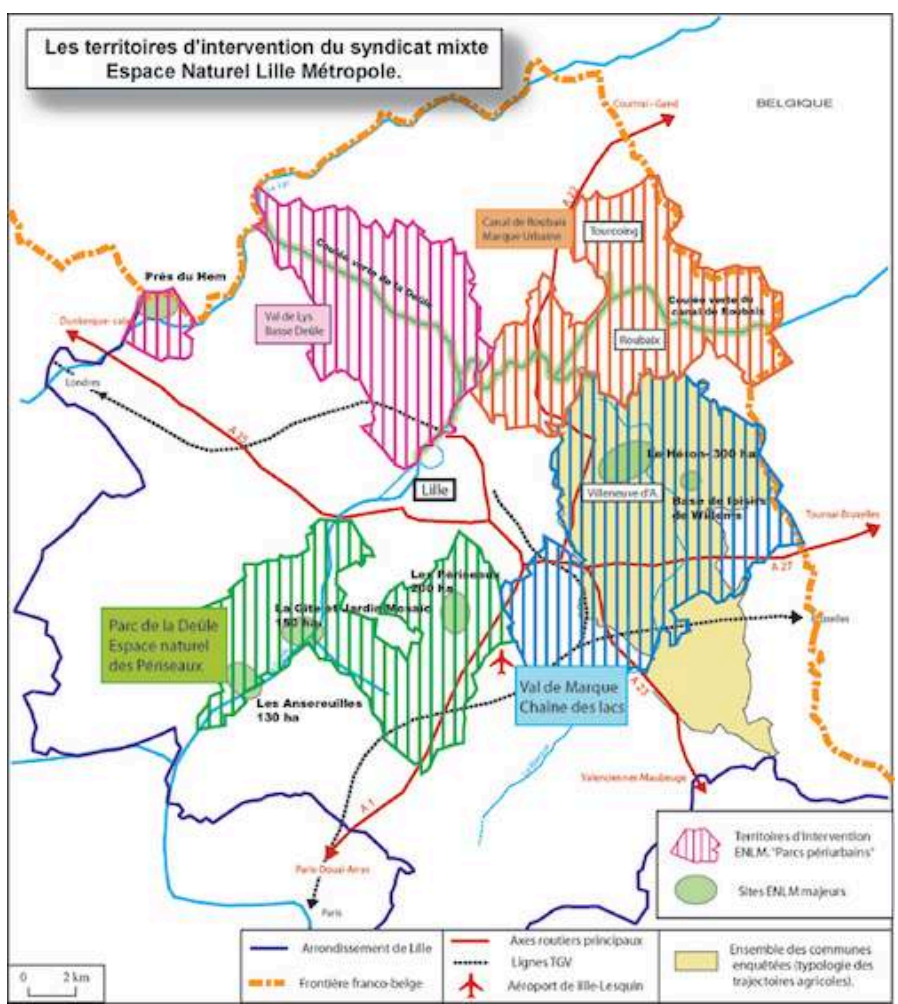

\section{L'émergence d'une nouvelle stratégie de gestion des « espaces naturels » périurbains}

Le projet d'intégrer la "nature » dans le territoire de la cité, à Lille, procède d'une volonté de répondre à trois urgences : environnementale (protéger les ressources en eau), paysagère (création d'un réseau maillé d'espaces verts) et économique (promouvoir la métropole - marketing territorial).

7 Les premières idées ou projets destinés à répondre à ces trois catégories d'objectifs se positionnaient aux dépens de, parfois contre, l'activité agricole.

\section{Les premiers projets : la nature aux dépens de l'agriculture}

8 Le schéma directeur d'aménagement et d'urbanisme de 1973 envisageait la création d'une vaste zone de loisirs au sud-ouest de la conurbation, s'étirant sur $16 \mathrm{~km}$ de long. Le premier projet d'un parc de la Deûle impliquait l'expropriation des agriculteurs sur 1200 hectares.

9 Les propositions de Bruno Bonduelle étaient plus radicales. Ce dernier est l'une des grandes figures du monde patronal Lillois. Il s'implique au début des années 1990 dans la gouvernance métropolitaine et publie sa Lettre aux 86 maires de Lille (Bonduelle, 1992) : la «ville à la manière du Nord" est à la campagne et cette campagne, il faut l'introduire. L'espace agricole est cependant décrit «mité », morcelé, défiguré. Bruno Bonduelle propose d'offrir aux agriculteurs deux fois l'indemnité d'éviction couramment pratiquée, ce à hauteur de 25000 hectares, pour transformer le cadre de 
vie des citadins : 10000 hectares auraient été consacrés aux maisons individuelles et à leurs jardins et 10000 hectares à la forêt.

10 L'intégration des « espaces naturels » dans le projet de territoire était unanimement envisagée, celle de l'activité et des espaces agricoles ne relevait alors aucunement de l'évidence.

\section{Le rapprochement des sphères urbaines et agricoles}

11 La reconnaissance urbaine de l'agriculture, en tant qu'activité à part entière du territoire de la ville et en tant qu'ensemble d'acteurs susceptibles de contribuer à la réalisation du projet métropolitain, s'opère au cours des treize années de révision du schéma directeur (1989-2002). À cette prise de conscience concourent la montée des notions de développement durable et de «bonne gouvernance» (Theys, 2003), mais aussi la fermeté des positions de la chambre d'agriculture.

\section{Les conflits entre la chambre d'agriculture et l'Agence de développement et d'urbanisme de Lille Métropole obligent à une prise en compte des impératifs fonciers agricoles}

12 Dans le contexte de révision du schéma directeur, deux face-à-face opposent la chambre d'agriculture à l'Agence d'urbanisme.

En 1994, en raison de différends portant sur l'ampleur des superficies agricoles classées constructibles, la chambre d'agriculture menace de déposer un recours et pousse ainsi à l'annulation de la délibération d'approbation. 315 hectares promis à l'urbanisation sont réaffectés. Surtout le principe de "progressivité » est adopté : « une partie des zones d'extension urbaine jusqu'alors consacrée à l'activité agricole doit être réservée à cet usage sur une durée d'au moins dix ans à partir de l'approbation du schéma directeur " (schéma directeur de développement et d'urbanisme de Lille Métropole, 1997).

14 En 2001, le conflit porte sur la localisation et les dimensions des zones d'activité. L'Agence d'urbanisme renonce à la création de zones d'activité de grande taille. La nouvelle version du document fait néanmoins l'objet d'un avis défavorable de la chambre d'agriculture. La communauté urbaine et la chambre d'agriculture organisent alors conjointement un séminaire intitulé « Le développement de la métropole lilloise et de son agriculture: quel partenariat?» (septembre 2002). Pierre Mauroy invite à " créer les conditions d'un partenariat entre la cité et le monde agricole ». L'agriculture est publiquement reconnue en tant qu'activité économique dont les exigences foncières sont légitimes, mais aussi en tant que producteur de «biens publics» (Poulot, 2008). L'offre émise par Pierre Mauroy doit rassurer les agriculteurs sur la nature des intentions urbaines et faciliter l'approbation du document d'urbanisme. Elle est motivée également par la réactivation d'un projet «Parc de la Deûle » qui désormais substitue à toute idée d'expropriation celle de "campagne cadre de vie " (PerrierCornet et Hervieu, 2002). Elle est justifiée enfin par le déploiement de systèmes de production agricole à « l'image citadine plus positive » (Fleury, 2005). 


\section{Les dynamiques de territorialisation de l'agriculture autorisent la définition de points de convergence des intérêts}

Le partenariat aujourd'hui engagé ne relève en effet pas de la seule évolution des représentations et des politiques urbaines. Dans un même temps, une large fraction des exploitants s'est engagée dans de véritables stratégies de reterritorialisation.

$\mathrm{Au}$ sein des quatorze communes enquêtées (cent exploitations) en périphérie est de l'agglomération lilloise (image 1), les deux tiers des agriculteurs tirent profit de la proximité urbaine : $16 \%$ par la mise en œuvre de systèmes de production spécialisés (image 2 - G II), 50 \% par l'offre de services aux particuliers (G III et G IV) (Rouget, 2008).

Figure 2. Typologie des trajectoires agricoles. Communes de la périphérie est de l'agglomération lilloise

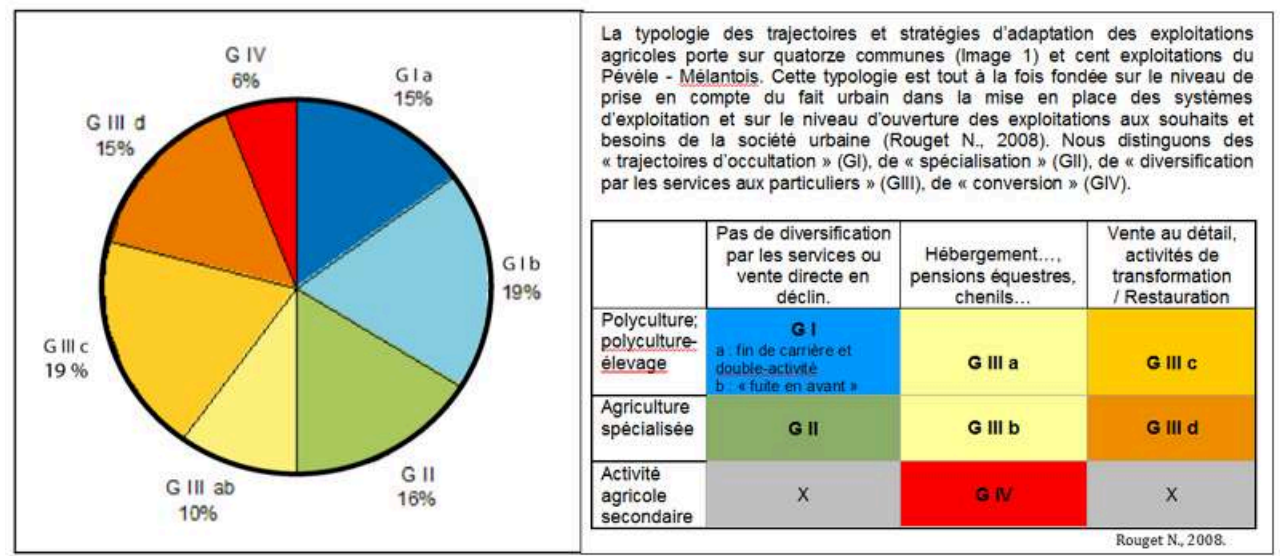

17 La grande diversité de l'offre agricole contribue directement à l'attention que les institutions urbaines portent aux revendications agricoles. L'agriculture dite de proximité est considérée par les aménageurs comme apte à produire une valeur ajoutée au territoire. Les produits cueillette ou achat à la ferme ne peuvent être le fait d'aucune entreprise et d'aucun espace autre qu'agricole.

Selon une perspective inversée, celle des chefs d'exploitation engagés dans des stratégies de territorialisation, l'agriculture se nourrit bel et bien de la ville et ne peut donc qu'accepter les contraintes inhérentes à la satisfaction des demandes urbaines (tant en termes d'ouverture des espaces, que de qualité paysagère). Par les trajectoires réalisées ou en cours, les agriculteurs rencontrés ont redéfini leurs liens à la société (Muller et al., 1989), développé un sentiment d'appartenance à un espace partagé. La communauté urbaine est dès lors un interlocuteur légitime.

Si le projet de territoire, dès les prémices de sa conception, impliquait l'intégration à la ville des « espaces naturels » périurbains, il excluait l'espace productif agricole. Par les conflits engagés en 1994-1995, puis en 2001-2002, et conformément aux principes du concept triomphant de développement durable, les décideurs ont perçu les hommes et les entreprises derrière le foncier. La mise en œuvre du "principe de progressivité ", l'attitude constructive de la chambre d'agriculture, le déploiement d'une agriculture de proximité contribuent à une mise en convergence des représentations du territoire. C'est par la promotion d'une " campagne ", certes cadre paysager, mais aussi conçue en tant qu'espace de production d'une activité agricole qui intègre à ses stratégies de 
développement la "publicisation» de son espace (Le Caro, et Kergreis, 2007), que convergent les intérêts. C'est pour l'émergence de cette « campagne » qu'est entrepris le partenariat ENLM - profession agricole.

\section{L'agriculture pour « partenaire »}

20 C'est surtout depuis 2003-2004 que l'agriculture des parcs périurbains est appelée à participer à la gestion des "espaces naturels métropolitains". Les premières expérimentations en matière de mise à contribution de l'agriculture ont cependant été conduites à compter des années 1990 au sein du périmètre de la Deûle.

\section{Le périmètre de la Deûle pour espace expérimental}

21 Dans le cadre des études lancées pour la révision du schéma directeur, le projet d'un Parc de la Deûle est réactivé en 1993. Ce projet n'implique plus l'expropriation des agriculteurs sur 1200 hectares et envisage une participation directe des exploitants. Les premières réactions des agriculteurs sont néanmoins négatives. La chambre d'agriculture investie dans un rôle d'interface s'engage dans une politique de sensibilisation des aménageurs d'une part, des exploitants agricoles d'autre part.

Un diagnostic agricole (Six, Vandercruyssen, 1994) fait prendre conscience aux aménageurs de la nécessité de travailler le plus possible dans le sens de mesures incitatives à des fins de responsabilisation des acteurs.

Par ailleurs, deux voyages d'étude sont proposés aux agriculteurs. La diversification et l'intégration paysagère en sont les thèmes. Une trentaine d'exploitants participe. La chambre met également en place en 1999 une session de formation intitulée "Se former pour valoriser son activité, son image ». Un groupe d'une dizaine d'agriculteurs émerge. Ce groupe constitue le point d'appui des collaborations engagées depuis.

\section{Les collaborations}

\section{Des accords tacites}

L'ENLM peut travailler avec les agriculteurs hors contractualisation, laissant la place à l'initiative, mais en accompagnant les exploitants. Lorsqu'un éleveur remplace par luimême du maïs en bord de rivière par des prairies, l'ENL finance la clôture.

Des collaborations à court terme 
Photo 1. Sur 11 hectares, une « spirale fleurie » enjambe le canal de Seclin

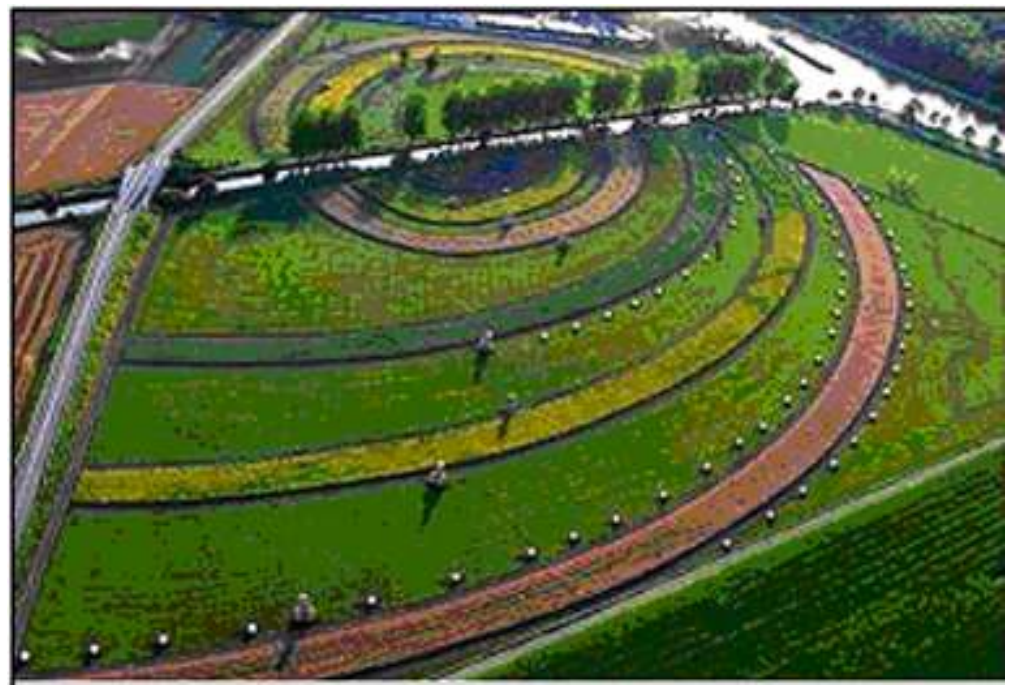

Source : LilleMétropolelnfo, nº 18, juin 2005 plus officielle ${ }^{3}$. Lille est alors capitale européenne de la culture et une vaste opération land art consacre l'ouverture du parc. Du 18 juin au 12 septembre, quarante hectares sur quatre sites sont dédiés à «l'articulture » et aux «folies agricoles » (image 3). Plusieurs agriculteurs participent à l'événement: mise à disposition de parcelles, contribution à la réalisation des visuels et à l'animation.

\section{Des opérations pérennes} synergie entre les exploitations. Les agriculteurs restent maîtres de l'offre, gardent l'initiative de leurs stratégies et de leurs trajectoires indépendamment de toute volonté extérieure. Ils ont néanmoins pour obligation d'adhérer à une démarche de qualité reconnue par la commission départementale d'orientation agricole. Les exploitants intégrés aux circuits bénéficient gratuitement de l'ensemble des outils de communication mis à leur disposition par le syndicat mixte. La diffusion de dépliants est assurée par les équipements ENLM et les offices du tourisme. Sur ces plaquettes, les fermes sont présentées, photographies à l'appui, et localisées sur une carte des sentiers pédestres.

Les programmes d'amélioration de la qualité paysagère et de promotion des trajectoires de territorialisation doivent contribuer à la pérennisation d'une large fraction des entreprises agricoles et donc emporter l'adhésion des chefs d'exploitation.

Projets de paysage, 8 | 2012 
L'objectif est de faire des émules, y compris au sein des systèmes a priori les moins à même de travailler avec la ville, pour les conduire à plus long terme à des ajustements et à des évolutions ${ }^{4}$.

Cependant, à Lille également, « les attentes suscitées par la participation locale » sont, dans une certaine mesure, « déçues » (Aubertin et al., 2008).

\section{Les limites du montage ville-agriculture}

31 La première des limites porte sur la volonté des agriculteurs à s'engager concrètement aux côtés de la ville dans le cadre d'une contractualisation. La problématique est également à relier au maintien, au sein du périmètre du schéma directeur, de communautés de communes indépendantes de la communauté urbaine de Lille. Enfin, par de nouvelles dynamiques de décrochage ${ }^{5}$, L'ENLM perd sa capacité à influer sur le devenir d'une part non négligeable des espaces agricoles.

\section{La difficile mobilisation des exploitants}

Dans le parc de la Deûle, alors que dix agriculteurs s'étaient initialement impliqués dans le programme d'intégration paysagère, seulement cinq projets ont abouti à l'issue de la première session. Dans le cadre des Circuits de fermes, seulement sept exploitations du parc de la Deûle (sur cinquante-deux) et quatre fermes du Val de Marque participent. Les agriculteurs de ce dernier espace considèrent très largement qu'il ne leur est pas nécessaire de s'insérer dans un réseau supplémentaire, pour lequel la signalétique fait par ailleurs défaut. Les clientèles sont établies. Les stages, réunions ou conditions d'agrément représentent des contraintes pour des exploitants dont les systèmes s'insèrent déjà pleinement dans le territoire urbain, mais qui restent peu désireux de « rendre des comptes ».

\section{Un territoire amputé}

L'existence d'une communauté de communes du Pays de Pévèle (image 4) met à mal la cohérence du Val de Marque. La communauté, maintenue en raison des craintes identitaires des élus, limite l'étendue des compétences de l'ENLM et exclut du partenariat les exploitations les plus à l'écart de l'agglomération. Pourtant, ces exploitations rencontrent davantage de difficultés pour établir ou fidéliser la clientèle des ateliers de diversification. Elles seraient, de ce fait, les plus susceptibles de participer aux programmes ENLM. 
Figure 3. Un territoire amputé : Val de Marque et communauté de communes du Pays de Pévèle

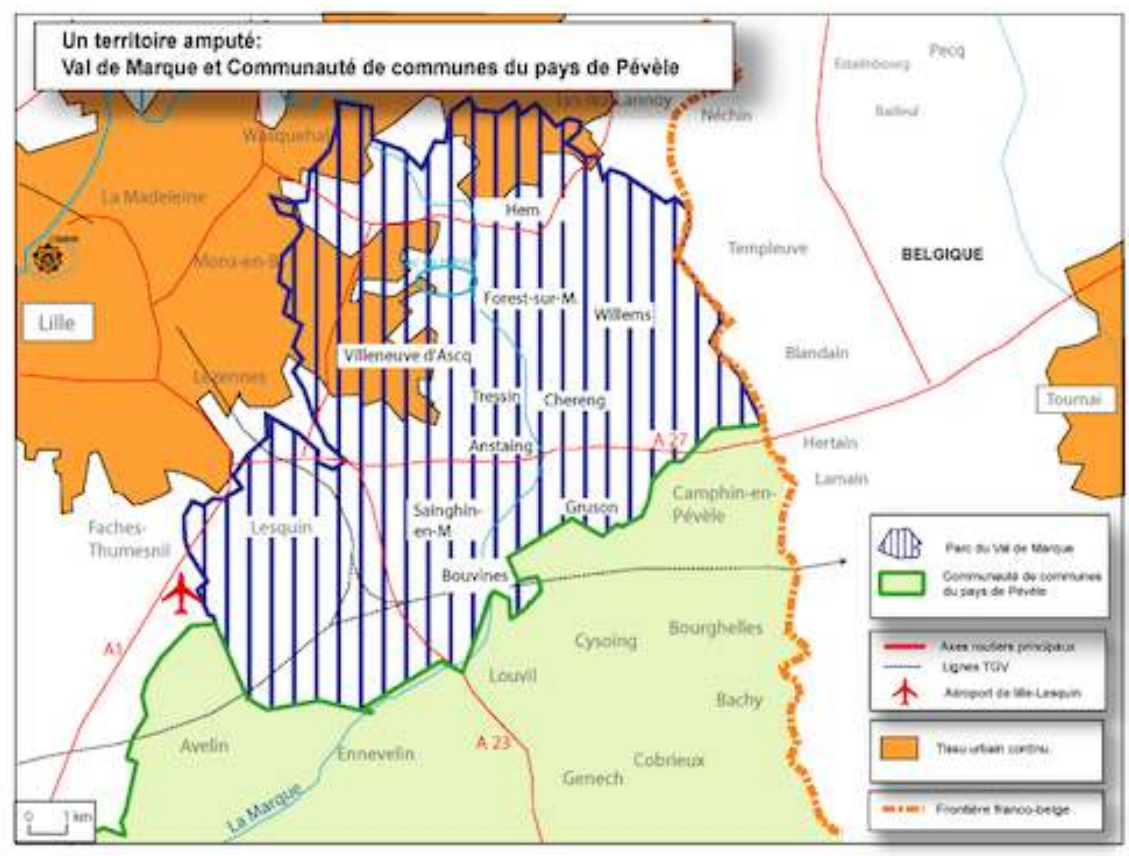

\section{Des dynamiques de décrochage}

décalage certain entre les aspirations urbaines et la réalité agricole. L'endive est un produit perçu comme identitaire. Éric Manouvrier (2004) souligne cependant la banalisation d'une production «désaisonnalisée » (semences hybrides, délocalisation des superficies mises en culture, utilisation de solutions hydroponiques).

À l'opposé de l'éventail des trajectoires agricoles, des exploitants développent des stratégies de conversion vers les activités de service (centres équestres notamment, logements, chenils). L'offre est standardisée. Surtout, les terres rendues disponibles alimentent les dynamiques d'agrandissement d'exploitations dont les sièges peuvent être plus largement ruraux. Ainsi d'importantes superficies sont louées à l'année à des agriculteurs belges dont les objectifs sont l'augmentation des volumes en pommes de terre et/ou la réalisation de leurs droits betteraviers (Rouget, 2008b). Au sein des dixsept communes françaises enquêtées à ce propos (image 5), une quinzaine d'agriculteurs wallons valorise 400 hectares. Ces terres ne satisfont plus qu'à des objectifs marchands exogènes, très éloignés des préoccupations paysagères ou d'animation de la communauté urbaine. Les stratégies de conversion et de mise à disposition des terres constituent autant de risques de désengagement des exploitants vis-à-vis des offres partenariales de l'ENML. 
Figure 4. Poids de la « présence belge » par commune

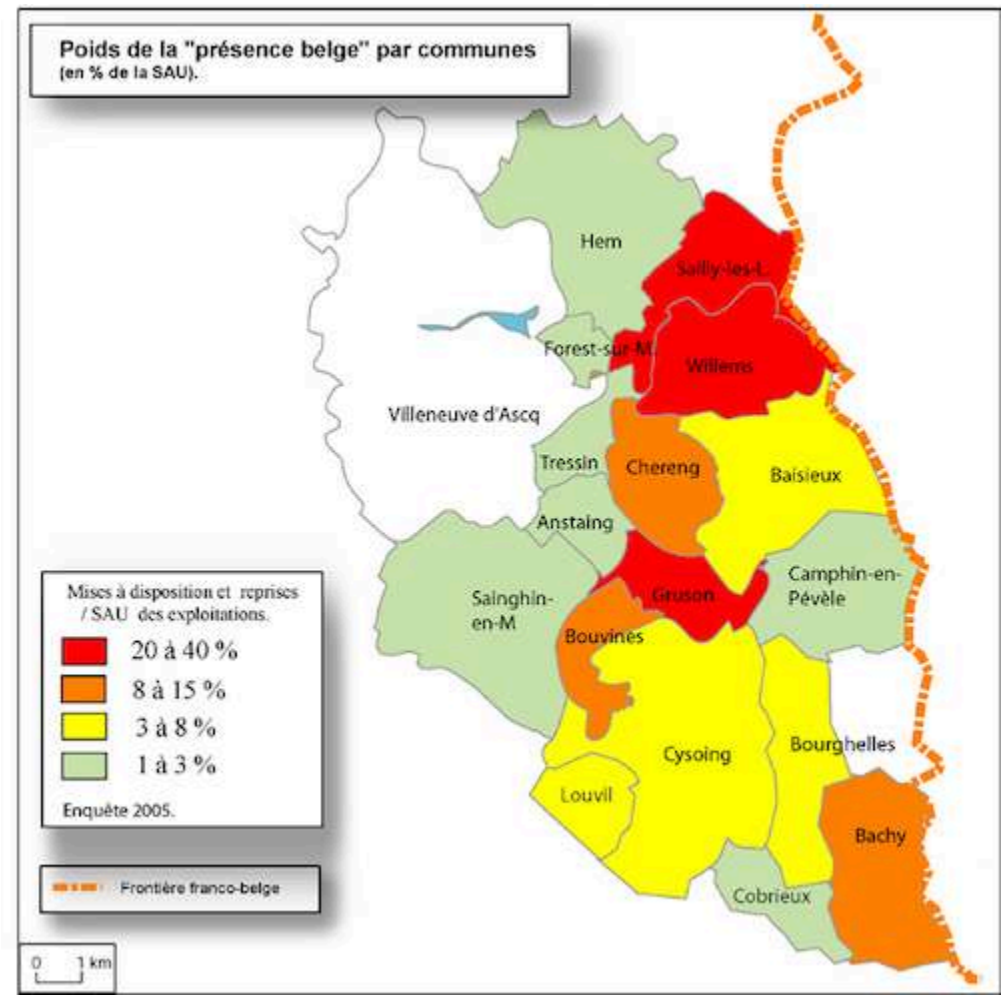

\section{Conclusion}

La mise en convergence des représentations et des intérêts entre décideurs urbains et acteurs agricoles résulte de la conjonction de deux dynamiques séparées mais synchroniques: simultanément à l'émergence d'une volonté urbaine de réinscription de la «nature » dans la cité, l'agriculture s'est insérée, par la mise en œuvre de stratégies de diversification et de valorisation de la proximité urbaine, dans le territoire de la ville. En satisfaisant aux revendications foncières des acteurs agricoles, les décideurs urbains certes permettent l'approbation du document d'urbanisme, mais aussi entérinent cette réinsertion de l'agriculture dans le territoire de la ville et dans « la logique urbaine » (Vidal, Fleury, 2009).

La réalisation des objectifs assignés aux parcs périurbains lillois passe largement par la mise en œuvre d'un véritable projet agriurbain. À l'agriculture incombe la charge de produire les "nouvelles campagnes ", cadres paysagers de nos périphéries urbaines qui à terme doivent concilier exigences environnementales et sociales. À la ville revient la responsabilité d'assurer la satisfaction des "besoins fonctionnels des exploitations " (Vidal, Fleury, 2009) et d'accompagner les trajectoires agricoles les plus aptes à contribuer à l'accomplissement du projet de territoire.

Un schéma directeur respectueux des impératifs fonciers agricoles, une agriculture diversifiée, l'implication forte des organisations professionnelles, des politiques de soutien aux exploitations agricoles fondées sur la contractualisation et la valorisation de l'existant ne constituent cependant pas des conditions suffisantes à la réussite d'un partenariat. Les réactions des agriculteurs face à un risque de mise en dépendance à l'égard des acteurs urbains sont vives. Le niveau d'engagement dans les Circuits de 
fermes est modéré. La réussite des stratégies agricoles de valorisation des opportunités urbaines dispense un certain nombre d'exploitations de l'offre "d'assistance réciproque " (Pierre Dhénin, directeur ENLM, réunion de présentation du partenariat ville-agriculture aux exploitants du Val de Marque, 2005) et par là même d'un droit de regard de la ville. Les concrétisations restent inachevées alors même que le pas à franchir pour un grand nombre de chefs d'exploitation est bien modeste.

Le temps dévolu à l'observation peut contribuer sans aucun doute à atténuer la crainte d'une confiscation de l'espace agricole par la ville. L'intégration de douze exploitations dans le Circuit de fermes du Val de Lys, dernier parc dans lequel le programme a été proposé, illustre le mûrissement du partenariat. Par ailleurs, un renforcement des intérêts perçus par le monde agricole (développement de la signalétique notamment) conduirait certainement des exploitants à dépasser leurs appréhensions.

La réussite d'un tel projet agriurbain de gestion participative des espaces implique des efforts permanents de sensibilisation, de formation, d'accompagnement et de relance. Celle-ci passe, fin 2010, par la nomination d'un conseiller communautaire délégué au monde agricole et à l'espace rural, et par la constitution d'un comité partenarial Lille Métropole/chambre d'agriculture.

\section{BIBLIOGRAPHIE}

Aubertin, C., Pinton, F., Rodary, E., «Le développement durable, nouvel âge de la conservation », dans Aubertin, C., Rodary, E. (sous la dir. de), Aires protégées espaces durables ?, Marseille, IRD/ Orstom, 2008, p. 17-27.

Biasi L. de, Pujol, D., Les Programmes agriurbains : un partenariat entre agriculteurs et collectivités, un nouveau mode de gouvernance, Paris, Iaurif-Driaf, 2005, $11 \mathrm{fasc} ., 51 \mathrm{p}$.

Bonduelle, B., Lettre aux 86 maires de Lille, Lille, Éditons La Voix du Nord, 1992, 245 p.

Fleury, A., « L'agriculture dans la planification de l'île-de-France : du vide urbain à la multifonctionnalité territoriale ", Les Cahiers de la multifonctionnalité, n 8, 2005, p. 33-46.

Le Caro, Y., Kergreis, S., « L'espace agricole comme espace public », dans Le Caro, Y., Madeline, P., Pierre, G. (sous la dir. de), Agriculteurs et Territoires. Entre productivisme et exigences territoriales, Rennes, PUR, 2007, p. 231-250.

Manouvrier, E., «L'endive, un produit de terroir du Nord-Pas-de-calais ? », Ruralia, 2004-2015, URL : https://journals.openedition.org/ruralia/1031.

Muller, P., Faure, A., Gerbaux, F., Les Entrepreneurs ruraux, Paris/Grenoble, L'Harmattan/ Université des sciences sociales de Grenoble, 1989, 189 p.

Perrier-Cornet, P., Hervieu, B., « Les transformations des campagnes françaises : une vue d'ensemble ", dans Perrier-Cornet, P. (sous la dir. de), Repenser les campagnes, La Tour-d'Aigues, L'Aube/Datar, 2002, p. 9-32. 
Poulot, M., « Des territoires de projet en périurbain : les programmes agriurbains », Historiens et Géographes, $n^{\circ} 403$, juillet août 2008, p. 159-172.

Poulot, M., Rouyres, T., « Refaire campagne en Île-de-France », Norois, $n^{\circ}$ 202, 2007/1, URL :

http://norois.revues.org/1631.

Rouget, N., « Des agriculteurs belges dans les "campagnes" de la périphérie lilloise : entre prédation et complémentarité ", dans Diry, J.-P. (sous la dir. de), Les Étrangers dans les campagnes, Clermont-Ferrand, Presses universitaires Blaise-Pascal, 2008(b), p. 349-360.

Rouget, N., « Les dynamiques agricoles dans les espaces urbains et périurbains. Diversification et stratégies d'adaptation des agricultures ", thèse de doctorat de géographie - université de Paris Ouest - Nanterre La Défense, 2008, 400 p. URL : http://tel.archives-ouvertes.fr/tel-00477267/fr/.

Six, M., Vandercruyssen, A.-C., « Projet du parc de la Deûle, diagnostic agricole économique et foncier ", chambre d'agriculture du Nord, Safer Flandres-Artois, 1994, 31 p.

Theys J., « La Gouvernance, entre innovation et impuissance », Développement durable et territoires, 2003, URL : http://developpementdurable.revues.org/1523

Vidal, R., Fleury, A., « La place de l'agriculture dans la métropole verte », Projets de paysage [En ligne], 09/02/2009, URL: http://www.projetsdepaysage.fr/fr/

la_place_de_1_agriculture_dans_la_metropole_verte.

\section{NOTES}

1. Le syndicat mixte Espace naturel Lille métropole (ENLM) s'est substitué le 1er octobre 2002 aux sept syndicats intercommunaux qui jusqu'alors étaient en charge, selon leurs problématiques propres, des missions de protection ou de requalification des espaces ou des patrimoines naturels. Le syndicat implique la communauté urbaine de Lille Métropole, les conseils général et régional et quarante communes de l'arrondissement.

2. L'ENLM opère directement sur des sites délégués par les collectivités locales: plan d'eau, espaces boisés, marais et zones humides... Aux opérations de requalification succèdent les équipements réglementant l'accès du public. C'est au sein des sites en gestion directe, mais aussi à l'échelle des périmètres définis par les territoires des quarante communes que l'agriculture des parcs est appelée à collaborer.

3. Une convention-cadre passée entre l'ENLM et la chambre d'agriculture précise les modalités de participation des agriculteurs et d'indemnisation. Une convention particulière établissant un échéancier précis et réalisant un état des lieux est par ailleurs signée entre l'ENLM et chaque agriculteur impliqué.

4. Signature en 2009 d'une convention entre Lille Métropole communauté urbaine et le Groupement des agriculteurs biologiques du Nord-Pas-de-Calais. L'objectif est d'accompagner le développement de l'agriculture biologique, notamment dans le secteur des champs captants du sud de Lille.

5. Formes de remise en cause du lien à la ville et/ou renoncement à toute - ou partie de l'activité de mise en culture par une fraction notable des chefs d'exploitation (image 2, G IV). 


\section{RÉSUMÉS}

Les agriculteurs de l'arrondissement de Lille sont appelés à partager, en tant qu'acteurs du territoire, la responsabilité du cadre paysager et environnemental de la métropole lilloise. L'objet de cet article est de comprendre comment et à quelles conditions se met en place une convergence d'intérêts entre acteurs $d u$ territoire. L'étude, fondée sur plusieurs séries d'entretiens conduits tant auprès des acteurs agricoles que des décideurs urbains, met en regard les trajectoires et dynamiques agricoles et le douloureux itinéraire du schéma directeur de Lille Métropole. L'exemple lillois pose également la question de l'efficience des expériences conduites en matière de participation locale: un schéma directeur respectueux des impératifs fonciers agricoles, une agriculture diversifiée et un partenariat fondé sur la contractualisation ne constituent pas des conditions suffisantes au plein accomplissement d'un projet agriurbain. Une gestion participative des espaces implique des efforts permanents d'accompagnement et de relance.

As long as they are playing their part in the development of the territory, the farmers of the area of Lille are assigned to share the responsability of the framework of the landsape and environment of their metropolis. The aim of the article is to understand how, and on which conditions, a convergence of interests between farmers and urban planners can be set up. The study, founded on several sets of discussions, brings face to face the agricultural paths and dynamics, together with the distressing route of the development plan of the area of Lille. Thanks to the example of Lille, we can also wonder how efficient the experiments led in the local participation are: a development plan respectful of the landed and agricultural imperatives, together with a diversified agriculture and a partnership based on the contractualisation don't constitute sufficient conditions to the full achievement of an agriurban project. A participative management of the land involves some continuous efforts of accompaniment and relaunching.

\section{INDEX}

Mots-clés : ville durable, agriculture, parcs périurbains, gestion participative, Lille

Keywords : sustainable city, agriculture, periurban parks, participative management, Lille (France)

\section{AUTEUR}

\section{NICOLAS ROUGET}

Maître de conférences en géographie, à l'université Lille Nord de France - CALHISTE (EA 4343) université de Valenciennes et du Hainaut-Cambrésis.

n.rouget[at]aposte[dot]net 